\title{
Improved prediction of mortality by combinations of inflammatory markers and standard clinical scores in patients with acute-on-chronic liver failure and acute decompensation
}

Henning Grønbæk ${ }^{1}$, Holger Jon Møller ${ }^{2}$, Fauzi Saliba ${ }^{3}$, Stefan Zeuzem ${ }^{4}$ Agustin Albillos ${ }^{5}$, Xavier Ariza ${ }^{6}$, Isabel Graupera ${ }^{6}$, Elsa Solà ${ }^{6}$, Alex Amoros ${ }^{7}$, Marco Pavesi ${ }^{7}$, Lars Bossen ${ }^{1}$, Rajiv Jalan ${ }^{8}$, ${ }^{*}$ Pere Gines ${ }^{6}$, *Vicente Arroyo ${ }^{7}$, on behalf of the CANONIC investigators, EASL CLIF Consortium.

1. Department of Hepatology \& Gastroenterology, Aarhus University Hospital, Aarhus, Denmark

2. Department of Clinical Biochemistry, Aarhus University Hospital, Aarhus, Denmark

3. Centre Hépato-Biliaire, AP-HP Hôpital Paul Brousse, INSERM, Unité 1193, Villejuif, France.

4. Medical Clinik I, Department of Internal Medicine, J.W. Goethe University Hospital, Frankfurt/Main, Germany.

5. Department of Gastroenterology, Hospital Universitario Ramón y Cajal, IRYCIS, Universidad de Alcalá, CIBEREHD, Madrid, Spain

6. Liver Unit, Hospital Clínic de Barcelona, University of Barcelona; Institut d'Investigacions Biomèdiques August Pi i Sunyer (IDIBAPS); Centro de Investigación Biomédica en Red de Enfermedades Hepáticas y Digestivas (CIBEReHD), Barcelona, Spain.

7. European Foundation for the study of Chronic Liver Failure (EF-CLIF), Barcelona, Spain.

8. UCL Institute for Liver and Digestive Health, University College London, United Kingdom.

*Shared last co-authorship

Corresponding author:

Henning Grønbæk, professor,MD,PhD

Department of Hepatology \& Gastroenterology,

Aarhus University Hospital,

99 Palle Juul-Jensens Boulevard,

DK-8200 Aarhus, Denmark.

Tel.:+4521679281.

E-mail address:Henngroe@ @rm.dk

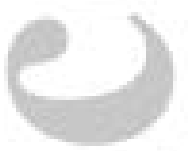

This article has been accepted for publication and undergone full peer review but has not been through the copyediting, typesetting, pagination and proofreading process which may lead to differences between this version and the Version of Record. Please cite this article as doi: 10.1111/jgh.15125 


\section{Conflict of interest:}

The study was supported by the European Foundation for the Study of Chronic Liver Failure (EF Clif). EF-Clif receives unrestricted donations from Grifols and Cellex Foundation and is partner or contributor in several EU Horizon 2020 program projects. EF-Clif and Cellex Foundation are non-profit private organizations. The funders had no influence on study design, data collection and analysis, decision to publish or preparation of the manuscript. The study also obtained funding from The Danish Strategic Research Council (TRAIN 10-092797); HG received funding from the NOVO Nordisk Foundation and "Savværksejer Jeppe Juhl og Hustru Ovita Juhls mindelegat". The remaining authors disclose no conflicts of interest.

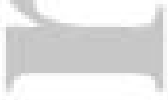

Abbreviations: ACLF (Acute-on-Chronic Liver Failure), AD (Acute Decompensation), MR (Mannose receptor), NGAL (neutrophil gelatinase associated lipocalin), DAMPs (Damage associated molecular patterns), PAPMs (Pathogen associated molecular patterns), HNA (redox state of circulating albumin)

Electronic word count: 3270

Number of tables and figures: 5 tables, 2 figures

\section{Abstract \\ Background and aim: Acute-on-chronic liver failure (ACLF)}

as a sinister prognosis and there is a need for accurate biomarkers and scoring systems to better characterize ACLF patients and predict prognosis. Systemic inflammation and renal failure are hallmarks in ACLF disease development and progression. We hypothesized that the combination of specific inflammatory markers in combination with clinical scores are better predictors of survival than the originally developed CLIF-C acute decompensation (AD) and CLIF-C ACLF scores.

Methods: We re-evaluated all previously measured inflammatory markers in 522 patients from the CANONIC study, 342 without and 180 with ACLF. We used the Harrell's C-index to determine the best marker alone or in combination with the original scores and calculated new scores for prediction of mortality in the original CANONIC cohort.

Results: The best markers to predict 90-day mortality in patients without ACLF were the plasma macrophage activation markers soluble (s)CD163 and mannose receptor (sMR). 
Urinary neutrophil gelatinase associated lipocalin (UNGAL) and SCD163 were predictors for 28-day mortality in patients with ACLF. The new developed CLIF-C AD+sMR score in patients without ACLF improved 90-days mortality prediction compared to the original CLIFC AD score (C-index 0.82(0.78-0.86) vs. 0.74(0.70-0.78, P=0.004). Further, the new CLIF-C ACLF+sCD163+UNGAL improved the original CLIF-C ACLF score for 28-days mortality (0.85(0.79-0.91) vs. 0.75(0.70-0.80), $\mathrm{P}=0.039)$.

Conclusions: The capability of these inflammatory markers to improve the original prognostic scores in cirrhosis patients without and with ACLF points to a key role of macrophage activation and inflammation in the development and progression of AD and ACLF.

\section{Key words:}

Hepatic inflammation, Kupffer cell, ACLF, Cirrhosis

Acute decompensation, CD163, Mannose receptor, Neutrophil gelatinase associated lipocalin (NGAL)

\section{Introduction:}

Acute-on-chronic liver failure (ACLF) is a disease entity that may develop in patients with chronic liver disease. The patients present with increasing number of organ failures and therefore hold a sinister prognosis. Acute decompensation (AD) represents another spectrum of liver disease progression with development of complications to liver cirrhosis and an increased risk for the development of ACLF. The CLIF CANONIC study ${ }^{1}$ aimed to investigate primarily ACLF but also AD in patients with liver cirrhosis. From the CANONIC study prognostic clinical scores have been developed. The CLIF-C ACLF score is calculated by the CLIF-C organ failure score (CLIF-C OF score ${ }^{1}$ ), combined with age and the white blood cell (WBC) count ${ }^{2}$. The CLIF-C AD score is based on age, S-sodium, WBC, creatinine and INR 3 .

A hallmark of $\mathrm{AD}$ and especially ACLF development and progression is local and systemic inflammation. In cirrhotic patients without ACLF AD may develop as a consequence of bacterial translocation from the intestines and associated pathogen associated molecular patterns (PAMPs) with subsequent initially local intestinal inflammation followed by systemic inflammation. Further propagation may occur due to production of damage associated molecular patterns (DAMPs) in the liver and other organs ${ }^{4}$. ACLF is an acute incident developing in cirrhosis patients and most often caused by a precipitating event e.g. sepsis, alcoholic, viral or ischemic hepatitis, TIPS or surgical procedures accompanied by acute 
systemic inflammation ${ }^{1}$.

Both local and especially systemic inflammation are very important for both AD and ACLF, and the WBC is a key component of the prognostic CLIF-C AD and CLIF-C ACLF scores. During the last years a number of other markers of inflammation and liver disease severity has been investigated in ACLF and AD. Among these are macrophage activation markers soluble (s)CD163 and mannose receptor (sMR) ${ }^{5}$ and Neutrophil Gelatinase-Associated Lipocalin (NGAL) ${ }^{6,7}$. Plasma sCD163 is associated with inflammation and fibrosis in chronic viral hepatitis and non-alcoholic fatty liver disease (NAFLD) ${ }^{8,9}$; and both SCD163 and sMR levels are associated with portal hypertension and prognosis in patients with liver cirrhosis ${ }^{10-12}$. Further, significantly elevated sCD163 levels predict prognosis in patients with acute liver failure and severe alcoholic hepatitis ${ }^{13,14}$, which suggest macrophages to play a key role in liver disease severity, progression and prognosis. Recently we also demonstrated reduced sCD163 and sMR levels following successful intervention in viral hepatitis and NAFLD ${ }^{15-17}$. NGAL is produced in a number of organs and cell types and especially demonstrated in the granules of neutrophil leucocytes. NGAL can be measured in plasma and urine with elevated levels in acute and chronic kidney diseases ${ }^{18}$. Recently, NGAL has been suggested as a marker for inflammation in experimental liver injury models ${ }^{19,20}$ and a few studies have investigated NGAL in patients with liver diseases ${ }^{21-23}$.

From the published data on biomarkers and prediction of prognosis in patients with $A D$ and ACLF the macrophage activation markers SCD163 and sMR along with NGAL seem to be the most promising ${ }^{5,6}$. We aimed to further investigate these biomarkers to provide insights into prognosis and further mechanistic information on the pathogenesis of AD and ACLF. We hypothesized that sCD163, sMR and NGAL can serve as single markers for prognosis in cirrhotic patients with $\mathrm{AD}$ and ACLF, and that new developed scores improve the prognostic capability compared to the original CLIF-C AD and CLIF-C ACLF scores.

\section{Methods:}

We included 522 patients from the CANONIC study where biomarkers were investigated in all patients and published during the past years. The biomarkers included macrophage activation markers, sCD163 and sMR ${ }^{5}$, plasma and urine NGAL ${ }^{6}, 29$ cytokines focused on plasma TNF, interleukin 6 (IL-6), IL-8, IL-10 ${ }^{24}$, the redox state of circulating albumin (HNA2), a marker of systemic oxidative stress, and plasma renin and copeptin ${ }^{24}$, as markers of systemic circulatory function, along with the plasma IL-1 receptor (IL-1R) ${ }^{25}$.

The CANONIC study was a multicenter study aimed at evaluating the frequency, 
characteristics, and outcome of ACLF in patients admitted to hospital for acute decompensation of cirrhosis in 29 liver units from 8 European countries. In the current study, ACLF was defined according to the criteria of the CANONIC study ${ }^{1}$, which are based on presence of organ failure(s) as defined according to CLIF-C SOFA score. Briefly, patients with ACLF were those with either: 1) single kidney failure; 2) single liver, coagulation, circulatory or respiratory failure associated with serum creatinine levels between 1.5 and $<2 \mathrm{mg} / \mathrm{dl}$ and/or hepatic encephalopathy grades I or II; 3) single cerebral failure (hepatic encephalopathy grades III or IV) associated with serum creatinine ranging from 1.5 and $<2 \mathrm{mg} / \mathrm{dl}$; or 4 ) two or more organ failures.

Out of the 1343 patients enrolled in the CANONIC study, 684 had both urine and plasma samples at the time of inclusion, and NGAL was measured as previously described ${ }^{6}$. Patients with urinary tract infection at the time of urine collection were excluded. Macrophage activation markers, sCD163 and sMR, were measured in 853 out of 1343 included patients in the CANONIC study as previously described ${ }^{5}$. Proinflammatory cytokines were measured in 522 patients, 237 with ACLF, as previously described ${ }^{24}$. We planned to investigate and compare all previously analyzed biomarkers in the same analysis and therefore restricted the present dataset to the 522 patients from the CANONIC study where all variables were present (342 patients with AD and 180 with ACLF).

For final evaluation and prediction we investigated the newly developed scores to the highest number of subjects included in the CANONIC study and where we had all available data ( $\mathrm{n}=853$ for $\mathrm{sMR}$ and $\mathrm{n}=129$ for $\mathrm{sCD} 163$ and UNGAL) for AD and ACLF prediction, respectively.

Informed consent in writing was obtained from each patient and the study protocol conformed to the ethical guidelines of the 1975 Declaration of Helsinki, as reflected in approval by the institutional review committee of participating centers.

\section{Statistics:}

From the above manuscripts we focused on inflammatory markers and cytokines showing significant associations with ACLF and AD prognosis and with data on all biomarkers in a cohort of 522 patients. Discrete variables are shown as counts (percentage) and continuous variables as mean (SD). Non-normally distributed variables are summarized by the median (interquartile range; IQR) and were log-transformed for some statistical analyses and for graphical comparisons. In univariate statistical comparisons, the chi-square test was used for categorical variables, whereas the Student t-test was used for normally distributed continuous 
variables and the Wilcoxon signed rank test for continuous variables not normally distributed. Harrell's concordance index (C-index) was used to estimate the discrimination ability of all markers and the new scores ${ }^{26}$. As a proportional hazard competing risk (PH-CR) model was used, C-index values and the corresponding 95\% confidence intervals (CIs) were estimated treating the transplanted patients as censored at the end of the follow-up, assuming that none of them could die before ${ }^{27}$. Statistical comparisons of the C-index were carried out for the main study time-points using the integrated discriminating improvement statistics ${ }^{26}$. To corroborate the results observed, a confirmatory analysis was carried out by estimating the Area Under the ROC curve (AUROC).

\section{Results:}

Baseline characteristics for the two groups of patients investigated are presented in Table 1. The ACLF patients were more likely to have alcoholic liver cirrhosis but otherwise there was no difference in ethiology, age or gender between the two groups. Decompensation with ascites or subrogates, hepatic encephalopathy and bacterial infections were more frequent in ACLF patients. Similarly, more ACLF patients displayed organ failures from liver kidney, brain, coagulation, heart and lung than patients without ACLF. This also included kidney dysfunction and mild to moderate hepatic encephalopathy. Further, we observed the expected differences in laboratory values (bilirubin, INR, albumin, creatinine and sodium) between patients with and without ACLF.

The patients with ACLF had a higher MELD score than patients without ACLF and a CLIF-C ACLF score of 49 while patients without ACLF had a CLIF-C AD score of 53. Further, there were significant differences in 28 day and 90 days mortality between the groups.

In addition, we observed significant differences in all cytokines and biomarkers between patients without and with ACLF.

\section{CLIF-C AD score and biomarkers for the prediction of 90-day prognosis in patients without ACLF.}

We calculated the 90-day Harrell's concordance index of MELD, CLIF-C-AD score and individual biomarkers alone and combined (Table 2). Both the MELD (0.70) and CLIF-C AD (0.73) scores performed well for the prediction of 90-day mortality. Only the macrophage activation markers sCD163 (0.70) and sMR (0.74) performed similarly to the CLIF-C AD score. Further, when SCD163 (0.77) or sMR (0.79) were added to the CLIF-C AD score there 
was a strong trend towards improvement of the CLIF-C AD score. A similar trend in improvement was observed for the CRP (0.76).

Since sMR showed the strongest prediction for 90-day prognosis in patients without ACLF we developed a new AD score based on age, creatinine, INR, WBC, sodium, and sMR (Table 3). This new CLIF-C AD+sMR (0.82) score was significantly better than the original CLIF-C AD (0.74) score in the prediction of the 90-day mortality in patients without ACLF. Further, sCD163 did not improve the prognostic accuracy of the CLIF-C AD+sMR score and was therefore not included.

In Figure 1A we present AUROC for MELD, CLIF-C AD score and the new developed CLIF$\mathrm{C}-\mathrm{AD}+\mathrm{sMR}$ score in all patients without ACLF from the CANONIC study, and the new score (0.82) was a better predictor than the original CLIF-C AD (0.76) score, $\mathrm{P}=0.005$.

Interestingly, the CLIF-C AD+sMR score (0.72(0.65-0.79) was also a good predictor for ACLF development $(\mathrm{n}=342)$ and performed better than the CLIF-C AD (0.69(0.62-0.77) and MELD 0.63(0.54-0.71) scores.

\section{CLIF-C ACLF score and biomarkers for the prediction of 28-days prognosis in patients with ACLF.}

We calculated the 28-day Harrell's concordance index of MELD, CLIF-C ACLF score and individual biomarkers alone and combined (Table 4). Both the MELD (0.69) and CLIF-C $\operatorname{ACLF}(0.76)$ scores performed well for the prediction of 28-days mortality. The best single biomarkers for prediction of mortality were UNGAL (0.76) and SCD163 (0.70) and added to the CLIF-C-ACLF score there was a trend for improvement in prediction especially for UNGAL (0.83).

These two parameters were included in the development of a new CLIF-C ACLF score for the prediction of 28-days mortality (Table 5). First, we calculated a CLIF-C ACLF+UNGAL score (0.83) based on the CLIF-C OF score, age, WBC, and U-NGAL with a trend towards improvement compared to the original CLIF-C ACLF (0.75) score ( $\mathrm{P}=0.068)$. Next, we added sCD163 to the scores and the CLIF-C ACLF+sCD163+UNGAL (0.85) significantly improved the 28-day prediction of mortality compared to the original CLIF-C ACLF (0.75) score. Thus, the addition of sCD163 improved the prognostic accuracy of CLIF-C ACLF+UNGAL score. In Figure 1B we present AUROC for MELD, CLIF-C ACLF score and the new developed CLIF-C-ACLF+sCD163+UNGAL score in all patients with ACLF from the CANONIC study $(n=129)$, and the new score (0.87) was a better predictor than the original CLIF-C AD (0.79) score $(\mathrm{P}=0.018)$. 
Of importance for 28-day mortality the novel CLIF-C ACLF+UNGAL+sCD163 score performed equally well in patients with $(0.86(0.76-0.95)$ and without $0.85(0.78-0.92)$ bacterial infections; and better than the CLIF-C ACLF (0.70(0.56-0.84) vs. 0.77(0.69-0.86)), CLIF-C OF (0.70(0.57-0.83) vs. 0.70(0.60-0.80)) and MELD (0.67(0.55-0.78) vs. 0.69(0.60-0.78)) for patients with and without bacterial infections, respectively. Similarly, the new score performed similarly well in patients with $(0.85(0.78-0.92)$ and without $(0.85(0.77-0.96)$ kidney dysfunction; and again better than the CLIF-C ACLF (0.77(0.68-0.86) vs. 0.73(0.60-0.85)), CLIF-C OF (0.72(0.63-0.81) vs. 0.73(0.60-0.87)) and MELD (0.71(0.62-0.80)) vs. 0.67(0.530.80)) for patients with and without kidney dysfunction, respectively.

Percent improvement in prediction of the new scores throughout 1-year follow-up with respect to MELD, CLIF-C ACLF and CLIF-C AD scores

The newly developed scores markedly improved the percentage of prediction at 28-, 90-, 180, and 365 days mortality compared to MELD and CLIF-C ACLF scores for patients with ACLF and for patients without ACLF compared to MELD and CLIF-C AD scores (Figure 2).

\section{Discussion:}

In the present study we re-investigated all previous investigated biomarkers predicting mortality in patients with cirrhosis from the CANONIC study. The main finding is the capability of the macrophage activation markers and UNGAL to improve the original prognostic scores in cirrhosis patients without (sMR) and with (sCD163 and UNGAL) ACLF, respectively. This points to a key role of macrophage activation and inflammation in the development and progression of $\mathrm{AD}$ and ACLF, which may be a potential future target strategy. In the present study we investigated patients included in the CANONIC study comprising both patients with cirrhosis and AD and ACLF, respectively. A number of predictors for morbidity and mortality has been described for patients with liver cirrhosis in general and includes the CP- and MELD scores, clinical information on previous decompensation episodes, as well as measures of portal hypertension and liver stiffness. Recently, an AD score derived from the CANONIC study for the prognosis of acute decompensation in patients with cirrhosis was proposed $^{3}$. For ACLF patients the CANONIC study revealed the CLIF-C ACLF score ${ }^{2}$. These scores improved prognostication beyond CP and MELD scores; however, there are still need for better scoring systems for selecting patients at highest risk for increased morbidity and mortality, and selection for specific treatments including liver transplantation. Importantly, we 
investigated central pathogenic biomarkers in these patient groups and were able to further improve the originally derived scoring systems.

Systemic inflammation is a hallmark in the development and progression of ACLF where an over activated immune response is accompanied by an inappropriate systemic inflammatory response with subsequent organ failures ${ }^{4,28}$. It is well known that cirrhotic patients have elevated levels of proinflammatory cytokines (e.g. TNF, Il-6), and immune cells from cirrhotic patients have more pronounced in vivo cytokine production after LPS stimulation compared to controls 29 . Especially, the innate immune system with monocytes and macrophages are involved in this cytokine production; and we showed that the macrophage activation markers SCD163 and SMR are elevated in association with liver diseases severity and portal hypertension $10,11,30$. This may suggest that with increasing liver disease severity there is increased macrophage activation, and when these macrophages are further activated by infection or inflammation they initiate and/or participate in an exaggerated immune response and cytokine storm leading to systemic inflammation, organ failure and ACLF. A recent study supported this with increased inflammatory marker levels in AD and where ACLF patients showed the largest number of abnormal markers suggesting "full-blown" systemic inflammation. Further, among AD-patients IL-8, IL-6, IL-1ra, HNA2 were independent predictors for 28-day progression defined as ACLF or death ${ }^{31}$. To further understand how inflammation is associated with metabolism and organ failure Moreau et al. investigated the blood metabolome in patients with cirrhosis, with and without ACLF. In ACLF patients the intensity of the blood fingerprint metabolite increased with ACLF severity and interestingly was significantly associated with macrophage activation and the elevated sCD163 and sMR levels. The metabolite represents different metabolic pathways including proteolysis and lipolysis; aminoacid catabolism; extra-mitochondrial glucose metabolism through glycolysis, pentose phosphate, and D-glucuronate pathways; depressed mitochondrial ATP-producing fatty acid beta-oxidation; and extramitochondrial amino acid metabolism ${ }^{32}$.

Triggers for this immune response may be PAMPs that react with pattern-recognition receptors including Toll-like receptors. Bacterial infections are very frequent in ACLF patients with spontaneous bacterial peritonitis and pneumonia accounting for approximately $30 \%$ of precipitating events ${ }^{1}$ while viral hepatitis is more frequent in Asian ACLF ${ }^{33}$. Another mechanism may be DAMPS during sterile inflammation caused by injured or dying hepatocytes ${ }^{4}$. The consequence of this over-activated immune response and production of inflammatory cytokines is impairment of the microcirculation in affected organs accompanied by impaired cell function and subsequent cell death ${ }^{4}$. This may further stimulate both local 
and liver macrophages in a viscous cycle but also lead to increased liver NGAL expression with increased plasma and urinary NGAL levels ${ }^{6}$.

Plasma sCD163 is a marker of macrophage activation with CD163 cleaved by the TACE/ADAM17 enzyme also responsible for shedding of TNF-alpha, which explain the strong correlation between sCD163 and TNF levels ${ }^{34}$. The sCD163 may represent both local production and systemic monocyte/macrophage activation and increased levels have been described in patients with sepsis and pneumonia with the highest levels in patients with chronic liver diseases ${ }^{35,36}$. As more than $80 \%$ of body macrophages resides in the liver sCD163 levels reflect to a high degree liver macrophage activation and we have previously demonstrated a gradient across the liver in patients with NAFLD and liver cirrhosis ${ }^{17,37}$. Similar findings have been presented for sMR ${ }^{30}$ including a gradient across the liver but without association to bacterial translocation ${ }^{11}$. However, the MR is in addition to macrophages also expressed on endothelial and dendritic cells, and the shedding is most likely caused by proteolytic cleavage ${ }^{38}$, and different from sCD163, which is shed by the TACE/ADAM enzyme ${ }^{34}$. From previous studies the highest SCD163 and sMR levels are found in patients with increasing liver disease severity with the highest levels in ACLF and ALF patients ${ }^{39}$. Importantly, circulating sCD163 and sMR are easily obtainable, stable during freezing and thawing, and both in house and commercial ELISA assays are available, which makes them ideal biomarkers for use also in daily clinical practice.

NGAL is a novel biomarker for inflammation and in liver diseases NGAL is stimulated by cell injury and regeneration as shown in acute and chronic liver injury models ${ }^{19,20,40}$. Plasma NGAL was higher in cirrhotic patients with impaired kidney function and in chronic viral hepatitis $C$ patients ${ }^{22,41}$. Gungor et al. showed higher plasma NGAL in cirrhotic patients with hepatorenal syndrome Type 1 (HRS-1) compared to HRS-2, and higher than controls and patients with cirrhosis and normal kidney function. Further, plasma NGAL was an independent predictor for mortality in these patients with an AUC of 0.82 similar to the MELD (AUC=0.81) and the $\mathrm{CP}$-score $(\mathrm{AUC}=0.80)^{42}$. Ariza et al. investigated $\mathrm{UNGAL}$ in patients with decompensated cirrhosis with acute kidney injury (AKI), HRS-1 and acute tubular necrosis (ATN) and found that UNGAL was best to predict ATN. Further, UNGAL was significantly elevated in ACLF patients and predicted ACLF with AUROC of 0.88 and UNGAL also predicted mortality in the whole group of patients investigated (AUROC $=0.88)^{23}$. These data were confirmed in the CANONIC study investigating plasma and urinary NGAL; and with higher UNGAL/g creatinine levels in ACLF patients, and UNGAL was an independent predictor for ACLF. Further, UNGAL independently predicted 28-day mortality and further 
improved the MELD score for mortality prediction while plasma NGAL was only a predictor in univariate analysis ${ }^{6}$. Interestingly, in liver biopsies Lipocalin-2 gene expression was highest in ACLF patients signifying intrahepatic production. A recent study partly confirmed these data with increased plasma NGAL in HBV ACLF patients with a poor prognosis and adding NGAL to the MELD score further improved prognostication from AUROC 0.76 (MELD) to 0.90 (MELD+NGAL) ${ }^{43}$. In further support, a translational study demonstrated elevated plasma NGAL in relation to liver disease severity and renal function along with higher levels in nonsurvivors compared to survivors ${ }^{44}$.

A limitation of UNGAL measurement is elevated levels in relation to urinary tract infections, which are frequent in cirrhotic and especially ACLF patients. Further, UNGAL may derive both from local production in the urinary tract or extraction from the circulation ${ }^{21}$. Further, in anuric patients UNGAL can obviously not be obtained, which makes the use of UNGAL as a biomarker less useful.

The main strength of the present study is the large number of well-characterized patients included in the CANONIC study at different European liver centers. This may also hold the risk of referral bias, as the hospitals involved in the study are primarily referral hospitals with highly specialized liver units. Another bias may be selection bias as all previous parameters were not investigated in the total number of patients from the original CANONIC cohort. However, sCD163 and sMR were investigated in 851 patients (185 with ACLF) and UNGAL in 716 patients (148 with ACLF) suggesting that the derived data and results are robust. Further, the study is in essence cross-sectional which may cause difficulties in interpretation of causality; however, in the original studies prospective examination of sCD163 and sMR showed that patients with stable or decreasing sCD163 levels had better prognosis compared to patients showing increased levels ${ }^{5}$.

We have provided novel scores based on specific biomarkers that improve the prediction for risk of death in patients with cirrhosis with $\mathrm{AD}$ and ACLF. However, assays for the biomarkers SCD163 and sMR are not yet internationally standardized, and typically measured by noncertified ELISA kits. To implement these in daily clinical practice will require standardization and preferably establishment of assays on automated analyses-platforms.

In conclusion, we demonstrated that specific markers of macrophage activation improved the original prognostic scores in cirrhosis patients without and with ACLF, which points to a key role of macrophage activation in the development and progression of AD and ACLF. In addition, UNGAL was a marker for ACLF mortality and the combination of NGAL and sCD163 improved the prognostic capability beyond the original CLIF-C ACLF score. 


\section{References}

[1] Moreau R, Jalan R, Gines P, et al. Acute-on-chronic liver failure is a distinct syndrome that develops in patients with acute decompensation of cirrhosis.

Gastroenterology. 2013; 144: 1426-37, 37 e1-9.

[2] Jalan R, Saliba F, Pavesi M, et al. Development and validation of a prognostic score to predict mortality in patients with acute-on-chronic liver failure. Journal of hepatology. 2014; 61: 1038-47.

[3] Jalan R, Pavesi M, Saliba F, et al. The CLIF Consortium Acute

Decompensation score (CLIF-C ADs) for prognosis of hospitalised cirrhotic patients without acute-on-chronic liver failure. Journal of hepatology. 2015; 62: 831-40.

[4] Claria J, Arroyo V, Moreau R. The Acute-on-Chronic Liver Failure Syndrome, or When the Innate Immune System Goes Astray. J Immunol. 2016; 197: 3755-61.

[5] Gronbaek H, Rodgaard-Hansen S, Aagaard NK, et al. Macrophage activation markers predict mortality in patients with liver cirrhosis without or with acute-onchronic liver failure (ACLF). J Hepatol. 2016; 64: 813-22.

[6] Ariza X, Graupera I, Coll M, et al. Neutrophil gelatinase-associated lipocalin is a biomarker of acute-on-chronic liver failure and prognosis in cirrhosis. Journal of hepatology. 2016; 65: 57-65.

[7] Fagundes C, Pepin MN, Guevara M, et al. Urinary neutrophil gelatinaseassociated lipocalin as biomarker in the differential diagnosis of impairment of kidney function in cirrhosis. J Hepatol. 2012; 57: 267-73.

[8] Kazankov K, Barrera F, Møller HJ, et al. Soluble CD163, a macrophage activation marker, is independently associated with fibrosis in patients with chronic viral hepatitis B and C. Hepatology (Baltimore, Md). 2014; 60: 521-30.

[9] Kazankov K, Barrera F, Moller HJ, et al. The macrophage activation marker sCD163 is associated with morphological disease stages in patients with non-alcoholic fatty liver disease. Liver international : official journal of the International Association for the Study of the Liver. 2016; 36: 1549-57.

[10] Gronbaek H, Sandahl TD, Mortensen C, Vilstrup H, Moller HJ, Moller S. Soluble CD163, a marker of Kupffer cell activation, is related to portal hypertension in patients with liver cirrhosis. Alimentary pharmacology \& therapeutics. 2012; 36: 173-80. [11] Laursen TL, Rodgaard-Hansen S, Moller HJ, et al. The soluble mannose receptor is released from the liver in cirrhotic patients, but is not associated with bacterial translocation. Liver international : official journal of the International Association for the Study of the Liver. 2017; 37: 569-75.

[12] Rode A, Nicoll A, Moller HJ, et al. Hepatic macrophage activation predicts clinical decompensation in chronic liver disease. Gut. 2013; 62: 1231-2.

[13] Moller HJ, Gronbaek H, Schiodt FV, et al. Soluble CD163 from activated macrophages predicts mortality in acute liver failure. Journal of hepatology. 2007; 47: 671-6.

[14] Sandahl TD, Gronbaek H, Moller HJ, et al. Hepatic macrophage activation and the LPS pathway in patients with alcoholic hepatitis: a prospective cohort study. Am J Gastroenterol. 2014; 109: 1749-56.

[15] Laursen TL, Wong GL, Kazankov K, et al. Soluble CD163 and mannose receptor associate with chronic hepatitis $B$ activity and fibrosis and decline with treatment. J Gastroenterol Hepatol. 2018; 33: 484-91. 
improvement of liver inflammation, fibrosis and metabolic liver function after successful direct - acting antiviral therapy of chronic hepatitis C. Journal of viral hepatitis. 2019; 27: 28-35.

[17] Kazankov K, Tordjman J, Moller HJ, et al. Macrophage activation marker soluble CD163 and non-alcoholic fatty liver disease in morbidly obese patients undergoing bariatric surgery. J Gastroenterol Hepatol. 2015; 30: 1293-300. [18] Makris K, Rizos D, Kafkas N, Haliassos A. Neurophil gelatinase-associated lipocalin as a new biomarker in laboratory medicine. Clin Chem Lab Med. 2012; 50: 1519-32.

[19] Xu MJ, Feng D, Wu H, et al. Liver is the major source of elevated serum lipocalin-2 levels after bacterial infection or partial hepatectomy: a critical role for IL6/STAT3. Hepatology (Baltimore, Md). 2015; 61: 692-702.

[20] Borkham-Kamphorst E, Drews F, Weiskirchen R. Induction of lipocalin-2 expression in acute and chronic experimental liver injury moderated by proinflammatory cytokines interleukin-1beta through nuclear factor-kappaB activation. Liver international : official journal of the International Association for the Study of the Liver. 2011; 31: 656-65.

[21] Hurry PK, Poulsen JH, Bendtsen F, Moller S. Neutrophil gelatinaseassociated lipocalin and cystatin $\mathrm{C}$ in cirrhosis and portal hypertension: Relations to organ extraction and dysfunction. J Gastroenterol Hepatol. 2017; 32: 473-81.

[22] Alhaddad OM, Alsebaey A, Amer MO, El-Said HH, Salman TA. Neutrophil Gelatinase-Associated Lipocalin: A New Marker of Renal Function in C-Related End Stage Liver Disease. Gastroenterology research and practice. 2015; 2015: 815484. [23] Ariza X, Sola E, Elia C, et al. Analysis of a urinary biomarker panel for clinical outcomes assessment in cirrhosis. PloS one. 2015; 10: e0128145.

[24] Claria J, Stauber RE, Coenraad MJ, et al. Systemic inflammation in decompensated cirrhosis: Characterization and role in acute-on-chronic liver failure. Hepatology. 2016; 64: 1249-64.

[25] Alcaraz-Quiles J, Titos E, Casulleras M, et al. Polymorphisms in the IL-1 gene cluster influence systemic inflammation in patients at risk for acute-on-chronic liver failure. Hepatology (Baltimore, Md). 2017; 65: 202-16.

[26] Harrell FE, Jr., Lee KL, Mark DB. Multivariable prognostic models: issues in developing models, evaluating assumptions and adequacy, and measuring and reducing errors. Stat Med. 1996; 15: 361-87.

[27] Fine J, Gray R. A proportional hazards model for the subdistribution of a competing risk. J Am Stat Assoc. 1999; 94.

[28] Arroyo V, Moreau R, Kamath PS, et al. Acute-on-chronic liver failure in cirrhosis. Nature Reviews Disease Primers. 2016; 2: 16041.

[29] Gandoura S, Weiss E, Rautou PE, et al. Gene- and exon-expression profiling reveals an extensive LPS-induced response in immune cells in patients with cirrhosis. $J$ Hepatol. 2013; 58: 936-48.

[30] Sandahl TD, Stoy SH, Laursen TL, et al. The soluble mannose receptor (sMR) is elevated in alcoholic liver disease and associated with disease severity, portal hypertension, and mortality in cirrhosis patients. PloS one. 2017; 12: e0189345. [31] Trebicka J, Amoros A, Pitarch C, et al. Addressing Profiles of Systemic Inflammation Across the Different Clinical Phenotypes of Acutely Decompensated Cirrhosis. Frontiers in immunology. 2019; 10: 476. 
inflammation-associated mitochondrial dysfunction as a potential mechanism underlying ACLF. Journal of hepatology. 2020; 72: 688-701.

[33] Sarin SK, Kumar A, Almeida JA, et al. Acute-on-chronic liver failure: consensus recommendations of the Asian Pacific Association for the study of the liver (APASL). Hepatol Int. 2009; 3: 269-82.

[34] Etzerodt A, Maniecki MB, Moller K, Moller HJ, Moestrup SK. Tumor necrosis factor alpha-converting enzyme (TACE/ADAM17) mediates ectodomain shedding of the scavenger receptor CD163. Journal of leukocyte biology. 2010; 88: 1201-5.

[35] Moller HJ, Moestrup SK, Weis N, et al. Macrophage serum markers in pneumococcal bacteremia: Prediction of survival by soluble CD163. Crit Care Med. 2006; 34: 2561-6.

[36] Kjaergaard AG, Rodgaard-Hansen S, Dige A, Krog J, Moller HJ, Tonnesen E. Monocyte expression and soluble levels of the haemoglobin receptor (CD163/sCD163) and the mannose receptor (MR/sMR) in septic and critically ill non-septic ICU patients. PloS one. 2014; 9: e92331.

[37] Holland-Fischer P, Gronbaek H, Sandahl TD, et al. Kupffer cells are activated in cirrhotic portal hypertension and not normalised by TIPS. Gut. 2011; 60: 1389-93.

[38] Nielsen MC, Andersen MN, Rittig N, et al. The macrophage-related biomarkers sCD163 and sCD206 are released by different shedding mechanisms. Journal of leukocyte biology. 2019; 106: 1129-38.

[39] Nielsen MC, Gantzel RH, Claria J, Trebicka J, H.J. M, Grønbæk H. Macrophage Activation Markers, CD163 and CD206, in Acute-on-Chronic Liver Failure. Cells. 2020; 9. [40] Borkham-Kamphorst E, van de Leur E, Zimmermann HW, et al. Protective effects of lipocalin-2 (LCN2) in acute liver injury suggest a novel function in liver homeostasis. Biochim Biophys Acta. 2013; 1832: 660-73.

[41] Gerbes AL, Benesic A, Vogeser M, Krag A, Bendtsen F, Moller S. Serum neutrophil gelatinase-associated lipocalin - a sensitive novel marker of renal impairment in liver cirrhosis? Digestion. 2011; 84: 82-3.

[42] Gungor G, Ataseven H, Demir A, et al. Neutrophil gelatinase-associated lipocalin in prediction of mortality in patients with hepatorenal syndrome: a prospective observational study. Liver international : official journal of the International Association for the Study of the Liver. 2014; 34: 49-57.

[43] Lu J, Lin L, Ye C, et al. Serum NGAL Is Superior to Cystatin C in Predicting the Prognosis of Acute-on-Chronic Liver Failure. Annals of hepatology. 2019; 18: 155-64. [44] Yoshikawa K, Iwasa M, Eguchi A, et al. Neutrophil gelatinase-associated lipocalin level is a prognostic factor for survival in rat and human chronic liver diseases. Hepatol Commun. 2017; 1: 946-56.

This article is protected by copyright. All rights reserved. 
Figure 1A: AUROC for the new developed score in all patients without ACLF

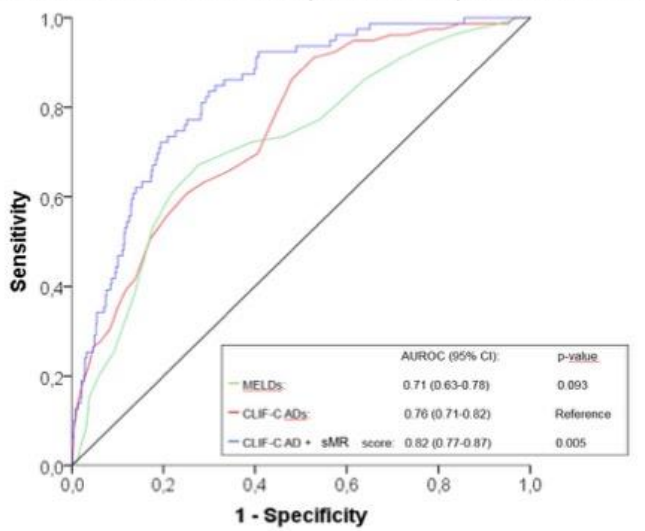

Figure 1B: AUROC for the new developed score in all patients with ACLF

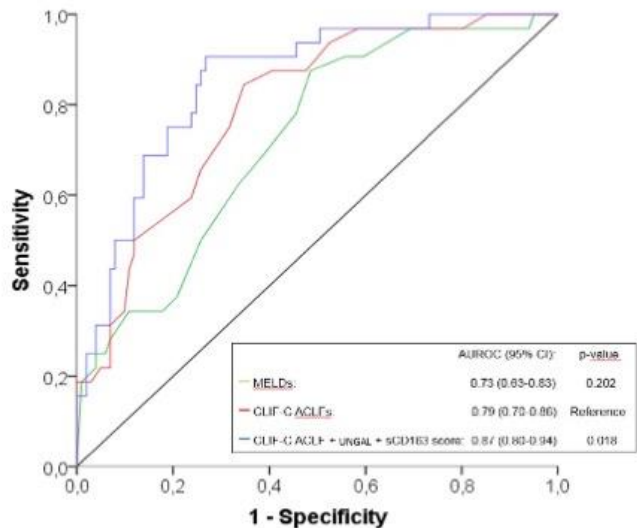

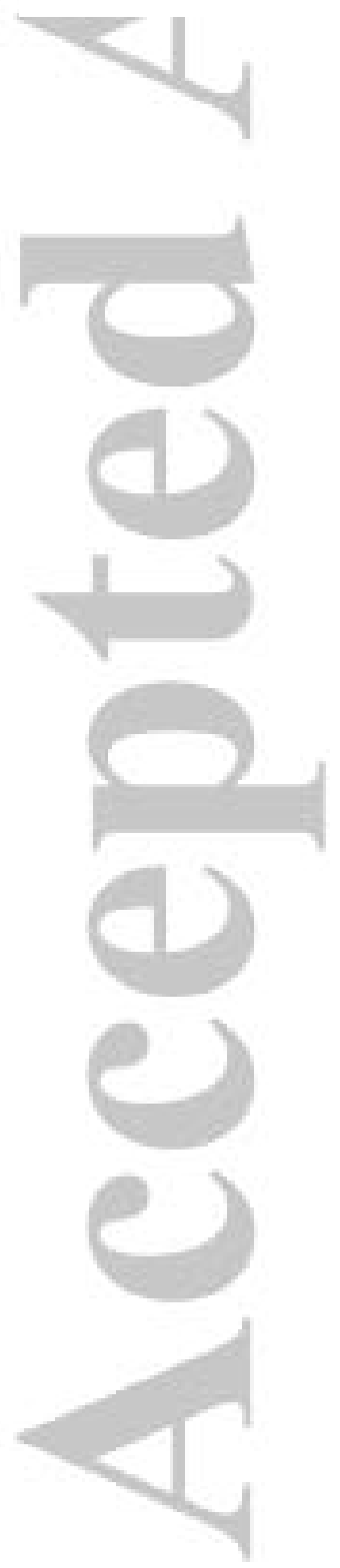

This article is protected by copyright. All rights reserved. 
Figure 3. Percent improvement in prediction for $28,90,180$ and 365 days mortality.

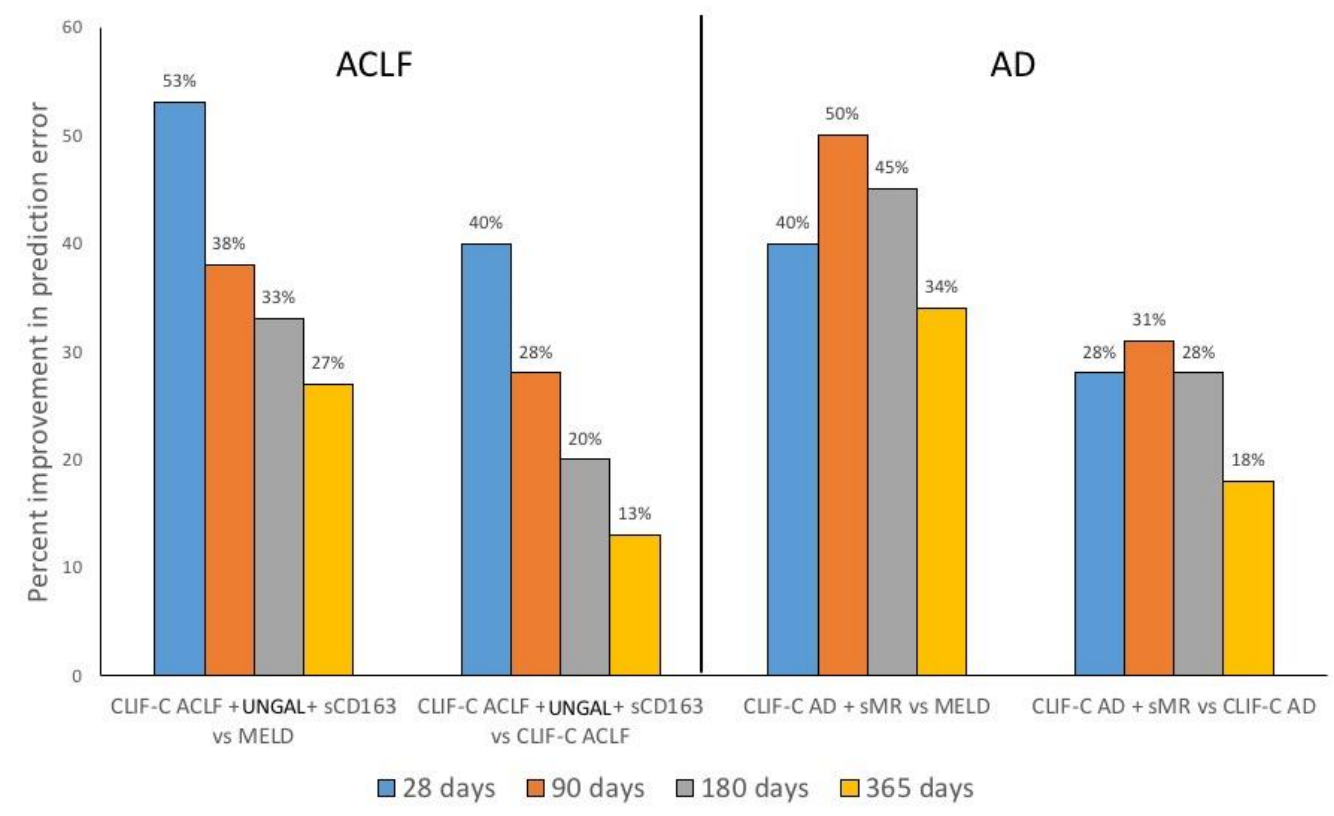

This article is protected by copyright. All rights reserved. 
Table 1. Baseline characteristics of patients without ACLF ( $n=342)$ and with ACLF $(n=180)$.

\begin{tabular}{|c|c|c|c|}
\hline Baseline characteristics & $\begin{array}{l}\text { No ACLF } \\
(\mathrm{N}=342)\end{array}$ & $\begin{array}{c}\text { ACLF } \\
(\mathrm{N}=180)\end{array}$ & $P$ value \\
\hline Age (years) & $56 \pm 12$ & $56 \pm 11$ & 0.814 \\
\hline Male & $227(66.4)$ & $117(65.0)$ & 0.753 \\
\hline \multicolumn{4}{|l|}{ Etiology } \\
\hline Alcohol (n, \%) & $157(48.9)$ & 99(58.2) & 0.049 \\
\hline $\operatorname{HCV}(n, \%)$ & $78(24.3)$ & $29(17.1)$ & 0.065 \\
\hline Alcohol + HCV (n, \%) & $29(9.0)$ & $20(11.8)$ & 0.337 \\
\hline Other (n, \%) & $57(17.8)$ & $22(12.9)$ & 0.167 \\
\hline Ascites with subrogates & $267(78.1)$ & $175(97.2)$ & $<0.001$ \\
\hline HE $(n, \%)$ & $90(26.3)$ & 105(58.3) & $<0.001$ \\
\hline GI bleeding (n, \%) & $65(19.0)$ & $20(11.1)$ & 0.020 \\
\hline Bacterial infection $(\mathrm{n}, \%)$ & $78(22.9)$ & $63(35.4)$ & 0.002 \\
\hline \multicolumn{4}{|l|}{ Organ Failures } \\
\hline Liver (n, \%) & $38(11.1)$ & $72(40.0)$ & $<0.001$ \\
\hline Kidney (n, \%) & - & $104(57.8)$ & - \\
\hline Brain (n, \%) & $8(2.3)$ & $35(19.4)$ & $<0.001$ \\
\hline Coagulation (n, \%) & $12(3.5)$ & $44(24.4)$ & $<0.001$ \\
\hline Cardiac (n, \%) & $5(1.5)$ & $29(16.1)$ & $<0.001$ \\
\hline Respiratory (n, \%) & $3(0.9)$ & $14(7.8)$ & $<0.001$ \\
\hline Kidney dysfunction ( $\mathrm{n}, \%)$ & $61(17.8)$ & $20(11.1)$ & 0.044 \\
\hline $\begin{array}{l}\text { Mild to moderate HE }(\mathrm{n}, \\
\%)\end{array}$ & $81(23.8)$ & $74(41.1)$ & $<0.001$ \\
\hline \multicolumn{4}{|l|}{ Laboratory values } \\
\hline Bilirubin (mg/dL) & $3.1(1.6-7.0)$ & $6.9(2.1-17.1)$ & $<0.001$ \\
\hline INR & $1.5(1.3-1.8)$ & $1.8(1.4-2.5)$ & $<0.001$ \\
\hline Albumin (g/dL) & $2.9(2.5-3.2)$ & $3.0(2.4-3.4)$ & 0.626 \\
\hline Creatinine $(\mathrm{mg} / \mathrm{dL})$ & $0.9(0.7-1.4)$ & $2.2(1.0-3.2)$ & $<0.001$ \\
\hline Sodium (mmol/L) & $136 \pm 6$ & $134 \pm 7$ & 0.010 \\
\hline \multicolumn{4}{|l|}{ Scores } \\
\hline MELDs & $17 \pm 6$ & $27 \pm 7$ & $<0.001$ \\
\hline CLIF-C ADs & $53 \pm 9$ & & - \\
\hline CLIF-C ACLFs & - & $49 \pm 9$ & - \\
\hline \multicolumn{4}{|l|}{ Mortality } \\
\hline 28 day & $22(6.4)$ & $48(26.7)$ & $<0.001$ \\
\hline 3 month & $55(16.1)$ & $72(40.0)$ & $<0.001$ \\
\hline \multicolumn{4}{|l|}{ Biomarkers } \\
\hline Leucocyte count $\left(10^{9} / \mathrm{L}\right)$ & $6.3(4.4-9.4)$ & $8.4(5.3-12.4)$ & $<0.001$ \\
\hline C-reactive protein $(\mathrm{g} / \mathrm{L})$ & $18(7-41)$ & $27(11-52)$ & 0.004 \\
\hline TNFa $(\mathrm{pg} / \mathrm{mL})$ & $20(14-29)$ & $29(17-43)$ & $<0.001$ \\
\hline IL6 (pg/mL) & $24(12-48)$ & $40(16-118)$ & $<0.001$ \\
\hline IL8 (pg/mL) & $42(22-84)$ & $89(41-177)$ & $<0.001$ \\
\hline IL10 $(\mathrm{pg} / \mathrm{mL})$ & $3.8(1.2-10.8)$ & $8.2(2.1-32.0)$ & $<0.001$ \\
\hline IL1Ra $(p g / m L)$ & $11(5-28)$ & $23(9-63)$ & $<0.001$ \\
\hline Urine NGAL (ng/ml) & $29(12-83)$ & $86(28-299)$ & $<0.001$ \\
\hline Plasma NGAL (ng/ml) & $139(102-213)$ & $235(147-448)$ & $<0.001$ \\
\hline $\mathrm{sCD} 163(\mathrm{mg} / \mathrm{L})$ & $8.8(5.2-12.6)$ & $13.8(7.9-19.0)$ & $<0.001$ \\
\hline $\mathrm{sMR}(\mathrm{mg} / \mathrm{L})$ & $0.8(0.6-1.1)$ & $1.0(0.7-1.5)$ & $<0.001$ \\
\hline HNA2 (\%) & $5.2(2.7-9.2)$ & $11.0(7.0-15.3)$ & $<0.001$ \\
\hline Renin (microlU $/ \mathrm{mL}$ ) & $72(20-274)$ & $121(34-353)$ & $<0.001$ \\
\hline $\mathrm{PCC}(\mathrm{pmol} / \mathrm{L})$ & $11(4-28)$ & $33(14-61)$ & $<0.001$ \\
\hline
\end{tabular}

This article is protected by copyright. All rights reserved. 
Table 2. Ninety-day Harrell's concordance index (C-index) of MELD, CLIF-C AD score and individual biomarkers (first column), and each biomarker added to CLIF-C ADs (second column) in patients without ACLF*.

\begin{tabular}{|l|c|c|c|}
\hline Variables & \multicolumn{2}{|c|}{ Mortality at 90 days } & \\
\hline MELD & $\begin{array}{c}\text { Individual } \\
\text { C-index(95\% IC) }\end{array}$ & $\begin{array}{c}\text { C-index(95\% IC) after } \\
\text { adding biomarkers to } \\
\text { CLIF-C ADs }\end{array}$ & $\begin{array}{c}\text { P-value between } \\
\text { CLIF-C ADs + } \\
\text { biomarkers vs CLIF- } \\
\text { C ADs }\end{array}$ \\
\hline CLIF-C AD & $\mathbf{0 . 7 0 ( 0 . 6 3 - 0 . 7 6 )}$ & & \\
\hline & $\mathbf{0 . 7 3 ( 0 . 6 6 - 0 . 7 9 )}$ & & \\
\hline Log(CRP) & $0.68(0.61-0.75)$ & $0.76(0.69-0.82)$ & 0.076 \\
\hline Log(TNFa) & $0.60(0.53-0.68)$ & $0.73(0.67-0.79)$ & 0.126 \\
\hline Log(IL6) & $0.65(0.55-0.70)$ & $0.72(0.66-0.78)$ & 0.901 \\
\hline Log(IL8) & $0.66(0.59-0.72)$ & $0.75(0.70-0.81)$ & 0.175 \\
\hline Log(IL10) & $0.57(0.49-0.65)$ & $0.71(0.65-0.78)$ & 0.470 \\
\hline Log(IL1Ra) & $0.57(0.49-0.64)$ & $0.73(0.66-0.79)$ & 0.374 \\
\hline Log(UNGAL) & $0.62(0.54-0.70)$ & $0.74(0.68-0.81)$ & 0.346 \\
\hline Log(PNGAL) & $0.63(0.55-0.71)$ & $0.73(0.67-0.80)$ & 0.157 \\
\hline Log(sCD163)* & $\mathbf{0 . 7 0 ( 0 . 6 4 - 0 . 7 7 )}$ & $\mathbf{0 . 7 7 ( 0 . 7 2 - 0 . 8 2 )}$ & $\mathbf{0 . 0 8 2}$ \\
\hline Log(sMR) & $\mathbf{0 . 7 4 ( 0 . 6 7 - 0 . 8 0 )}$ & $\mathbf{0 . 7 9 ( 0 . 7 4 - 0 . 8 4 )}$ & $\mathbf{0 . 0 7 2}$ \\
\hline Log(HNA2) & $0.66(0.59-0.73)$ & $0.73(0.67-0.79)$ & 0.251 \\
\hline Log(Renin) & $0.60(0.52-0.67)$ & $0.72(0.66-0.79)$ & 0.642 \\
\hline Log(Copeptin) & $0.64(0.57-0.72)$ & $0.73(0.66-0.80)$ & 0.860 \\
\hline
\end{tabular}

* Variable coefficients used for CLIF-C ADs were those originally described. SCD163 and sMR, which were the best markers predicting prognosis and increasing the accuracy of CLIF-C ADs, were included in the assessment of a new score for patients with AD. 
Table 3. Coefficients and c-index of the new score in AD patients at 90 days*.

\begin{tabular}{|c|c|}
\hline \multicolumn{2}{|c|}{ CLIF-C AD + sMR score = } \\
\hline \multicolumn{2}{|c|}{ Mortality at 90 days } \\
\hline CLIF-C AD & C-index $=0.74(0.70-0.78)$ \\
\hline \multicolumn{2}{|l|}{1} \\
\hline Variables & Coefficients \\
\hline Constant & +4 \\
\hline Age & 0.02 \\
\hline Log(Creatinine) & 0.11 \\
\hline $\log ($ INR $)$ & 1.96 \\
\hline $\log (W B C)$ & 0.75 \\
\hline Sodium & -0.02 \\
\hline Log(sMR) & 1.43 \\
\hline Readjusting & *10 \\
\hline \multicolumn{2}{|l|}{ 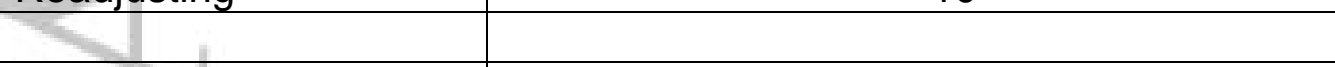 } \\
\hline C-index & $0.82(0.78-0.86)$ \\
\hline $\mathrm{P}(\mathrm{CLIF}-\mathrm{C} \mathrm{AD})$ & 0.004 \\
\hline
\end{tabular}

* Variable coefficients of CLIF-C ADs + sMR were recalculated due to the addition of the new marker. 
Table 4. Twenty-eight day Harrell's concordance index (C-index) of MELD, CLIF-C ACLF score and individual biomarkers (first column), and each biomarker added to CLIF-C ACLFs (second column) in patients with ACLF*.

\begin{tabular}{|l|c|c|c|}
\hline & \multicolumn{3}{|c|}{ Mortality at 28 days } \\
\hline Variables & C-index(95\% IC) & $\begin{array}{c}\text { C-index(95\% IC) } \\
\text { after adding } \\
\text { biomarkers to } \\
\text { CLIF-C ACLFs }\end{array}$ & $\begin{array}{c}\text { P-value between } \\
\text { CLIF-C ACLFs + } \\
\text { biomarkers vs } \\
\text { CLIF-C ACLFs }\end{array}$ \\
\hline MELD & $\mathbf{0 . 6 9 ( 0 . 6 2 - 0 . 7 6 )}$ & & \\
\hline CLIF-C ACLF & $\mathbf{0 . 7 6 ( 0 . 6 8 - 0 . 8 3 )}$ & $\mathbf{0 . 7 6 ( 0 . 6 8 - 0 . 8 3 )}$ & \\
\hline Log(CRP) & $0.57(0.49-0.66)$ & $0.75(0.67-0.83)$ & 0.346 \\
\hline Log(TNFa) & $0.55(0.47-0.63)$ & $0.77(0.70-0.84)$ & 0.496 \\
\hline Log(IL6) & $0.61(0.53-0.70)$ & $0.76(0.69-0.83)$ & 0.729 \\
\hline Log(IL8) & $0.65(0.58-0.72)$ & $0.77(0.70-0.83)$ & 0.416 \\
\hline Log(IL10) & $0.60(0.52-0.68)$ & $0.75(0.68-0.83)$ & 0.305 \\
\hline Log(IL1Ra) & $0.59(0.51-0.67)$ & $0.76(0.68-0.83)$ & 0.933 \\
\hline Log(UNGAL) & $\mathbf{0 . 7 6 ( 0 . 6 9 - 0 . 8 3 )}$ & $\mathbf{0 . 8 3 ( 0 . 7 6 - 0 . 8 9 )}$ & $\mathbf{0 . 0 7 6}$ \\
\hline Log(PNGAL) & $0.67(0.57-0.76)$ & $0.78(0.71-0.86)$ & 0.552 \\
\hline Log(sCD163) & $\mathbf{0 . 7 0 ( 0 . 6 3 - 0 . 7 8 )}$ & $\mathbf{0 . 7 9 ( 0 . 7 2 - 0 . 8 6 )}$ & $\mathbf{0 . 2 3 1}$ \\
\hline Log(sMR) & $0.66(0.59-0.74)$ & $0.76(0.69-0.83)$ & 0.370 \\
\hline Log(HNA2) & $0.58(0.50-0.66)$ & $0.78(0.72-0.85)$ & 0.264 \\
\hline Log(Renin) & $0.55(0.46-0.64)$ & $0.75(0.68-0.83)$ & 0.357 \\
\hline Log(Copeptin) & $0.63(0.54-0.72)$ & $0.77(0.69-0.85)$ & 0.474 \\
\hline
\end{tabular}

* Variable coefficients used for CLIF-C ACLFs were those originally described. Urine NGAL and SCD163, which were the best markers predicting prognosis and increasing the accuracy of CLIF-C ACLFs, were included in the assessment of a new score for patients with ACLF. 
Table 5. Coefficients and C-index of the new score in ACLF patients at 28 days*.

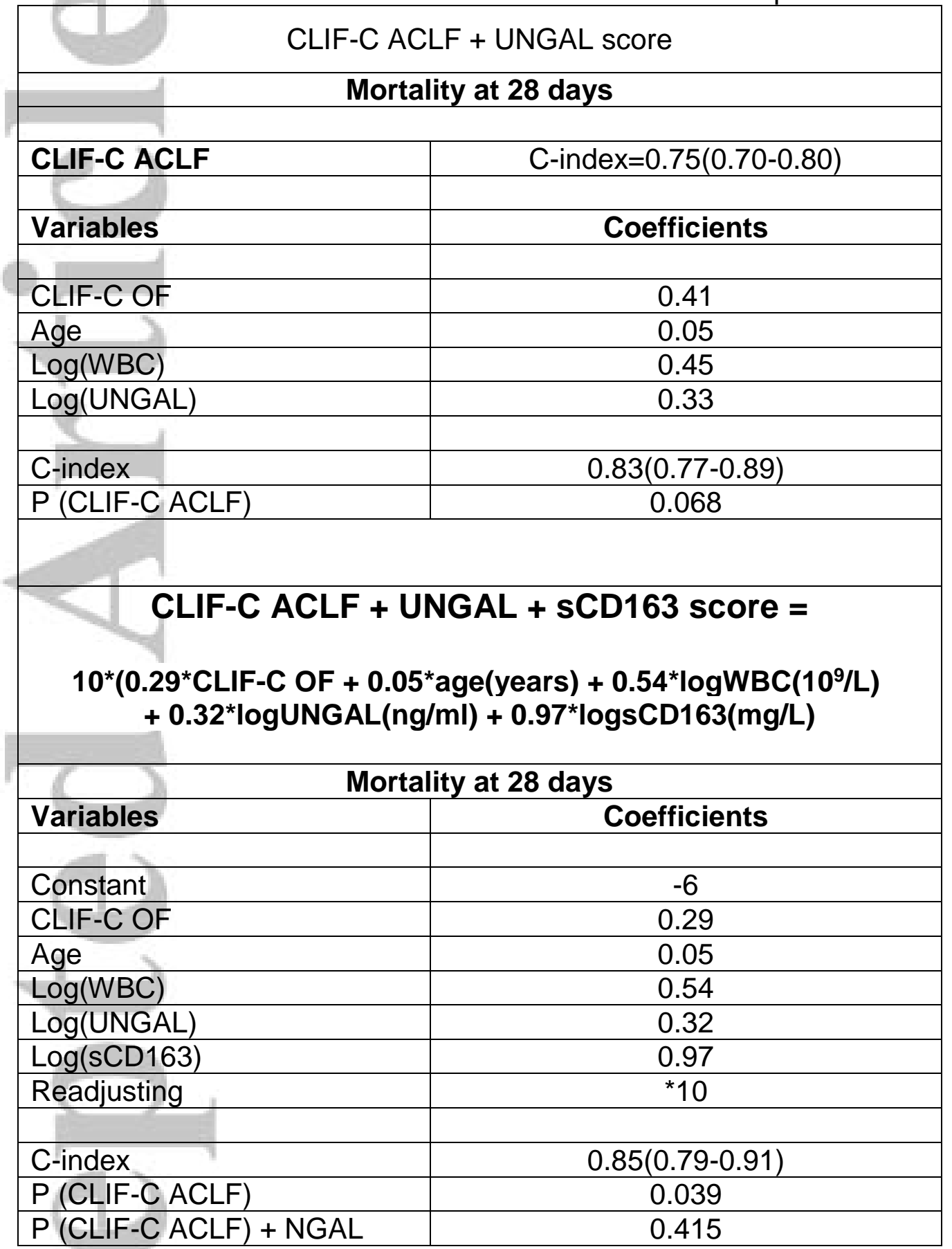

* Variable coefficients of CLIF-C ACLFs + UNGAL + SCD163 score were recalculated due to the addition of the new markers. 